\title{
Direct Observations of Retention Failure in Ferroelectric Memories
}

\author{
Peng Gao, Christopher T. Nelson, Jacob R. Jokisaari, Yi Zhang, Seung-Hyub Baek, \\ Chung Wung Bark, Enge Wang, Yuanming Liu, Jiangyu Li, Chang-Beom Eom, \\ and Xiaoqing Pan*
}

The ability to reversibly switch the spontaneous polarization with an applied electric field and a small critical domain size makes ferroelectric materials attractive for high-density nonvolatile memories. ${ }^{[1-4]}$ However, the written polarization often suffers from a gradual backswitching leading to data loss. ${ }^{[5-10]} \mathrm{At}$ larger scales this process can be attributed to a thermodynamic instability of the written polarization due to electrostatic or elastic energy from built-in electric fields ${ }^{[6,11-14]}$ and strain. ${ }^{[15,16]}$ However, we find that in writing nanosized domains, such as for memory bits in high bit-density storage, the domain wall itself can drive backswitching. Using high resolution in situ transmission electron microscopy we show that localized $180^{\circ}$ polarization switching in a $\mathrm{PbZr}_{0.2} \mathrm{Ti}_{0.8} \mathrm{O}_{3}$ (PZT) film initially forms domain walls along unstable planes due to the inhomogeneous electric field from the small switching electrode. After removal of the external field, they tend to relax to low energy orientations. In sufficiently small domains this process results in complete backswitching. Our results suggest that even thermodynamically favored domain orientations are still subject to retention loss, which must be mitigated by overcoming a critical domain size.

The polarization in ferroelectric oxides such as PZT results from an electric dipole moment created by relative displacements between cations and anions from their centrosymmetric

Dr. P. Gao,,$^{[+]}$Dr. C. T. Nelson,,$^{[+]}$J. R. Jokisaari, Dr. Y. Zhang,

Prof. X. Q. Pan

Department of Materials Science and Engineering

University of Michigan

Ann Arbor, Michigan 48109, USA

E-mail: panx@umich.edu

Dr. Y. Zhang

National Laboratory of Solid State Microstructures and Department

of Materials Science and Engineering

Nanjing University

Nanjing, 210093, P.R. China

Dr. S.-H. Baek, Dr. C. W. Bark, Prof. C.-B. Eom

Department of Materials Science and Engineering

University of Wisconsin-Madison

Madison, Wisconsin 53706, USA

Prof. E. G. Wang

International Center for Quantum Materials and School of Physics

Peking University

Beijing 100871, China

Y. M. Liu, Prof. J. Y. Li

Department of Mechanical Engineering

University of Washington

Seattle, WA 98195, USA

[+] P. G. and C. T. N. contributed equally to this work.

DOI: 10.1002/adma.201103983 positions. There are six nominally degenerate $<100>$ polarization directions in tetragonal PZT, but electric and strain fields will preferentially favor one or more orientations. The surface energy of the domain walls which separate regions with different polarizations are typically less than a few hundred $\mathrm{mJ} /$ $\mathrm{m}^{2} \cdot{ }^{[17,18]}$ This is sufficiently small in comparison to depolarizing fields and strain energy that ferroelectric films intrinsically adopt polydomain structures. However, this energy can become prohibitive for nanoscale domains since it scales with the surface area of the domain rather than its volume, and is well known for imposing a critical size barrier for the nucleation of new domains. In this work we find that a similar but larger size barrier is imposed for switching stability owing to the metastable domain wall orientations formed under the inhomogeneous applied electric field from a surface probe.

Local polarization switching was performed in a single crystal $100 \mathrm{~nm}$ thick (001) oriented $\mathrm{Pb}\left(\mathrm{Zr}_{0.2} \mathrm{Ti}_{0.8}\right) \mathrm{O}_{3}$ film grown on a $(110)_{\mathrm{O}} \mathrm{DyScO}_{3}$ substrate with an epitaxial $50 \mathrm{~nm}(110)_{\mathrm{O}} \mathrm{SrRuO}_{3}$ (SRO) conducting electrode ${ }^{[19,20]}$ (subscript O corresponds to orthorhombic indices). Small compressive strain from the $\mathrm{DyScO}_{3}$ substrate produces a $c$-axis oriented film, i.e., the polarization axis is normal to the film, yielding two possible polarization directions, upward to the free surface $\left(P_{[001]}\right)$ or downward to the substrate $\left(P_{[00 \overline{1}]}\right)$. Intrinsic electric fields arise internally from the polar surfaces (depolarizing fields), space charge layers, and injected charges, which can significantly influence the domain structure. In this case the SRO electrode partially screens the depolarizing fields but introduces a space charge layer in the form of a Schottky junction with a field along the film normal (E\| [001]) promoting an upward polarization $\left(P_{[001]}\right)$ at this interface. Highly localized polarization switching is produced by applying a bias between a scanning tungsten probe acting as top electrode and the SRO bottom electrode. An outof-plane electric field concentrated directly below the sharp tip promotes the local reversal of the polarization along the $z$-axis, i.e., $180^{\circ}$ switching, which occurs by a domain nucleation and growth mechanism. ${ }^{[21]}$ To capture switching at this scale, the real-time domain structure is characterized by in situ transmission electron microscopy (TEM). The increased spatial resolution of TEM over electronic ${ }^{[5,8-10]}$ or surface probe studies ${ }^{[6,7,15]}$ and the access to depth-dependent structural information provides a complete picture of the stable and metastable domain structure in the film during switching. ${ }^{[22-24]}$

The switching induced by a surface probe is characterized using piezoresponse force microscopy (PFM) and by in situ TEM in Figure 1. An out-of-plane PFM image (Figure 1a) shows the boundary between regions biased by the surface probe at $+17.5 V_{\mathrm{DC}}$ (yellow region) and $-17.5 V_{\mathrm{DC}}$ (red region). The 


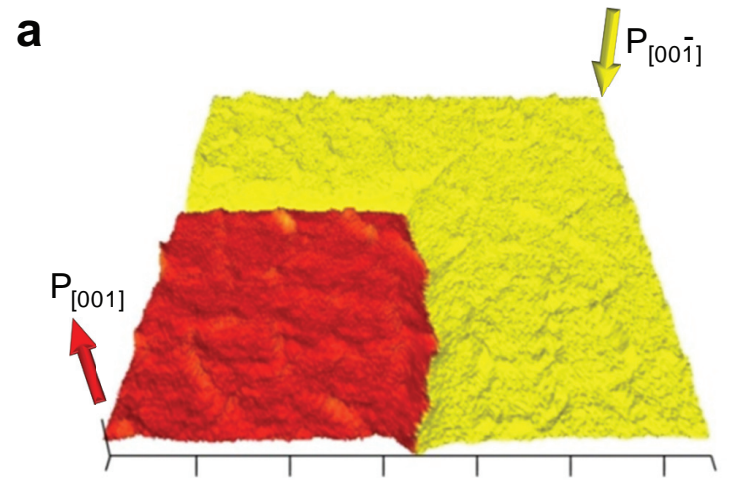

b

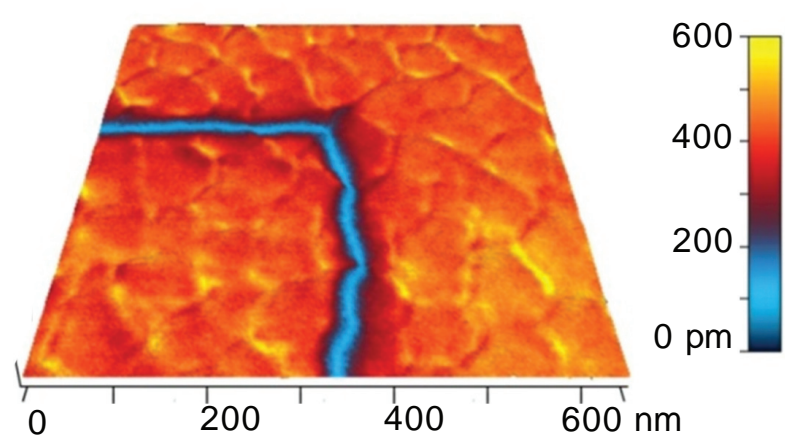

C

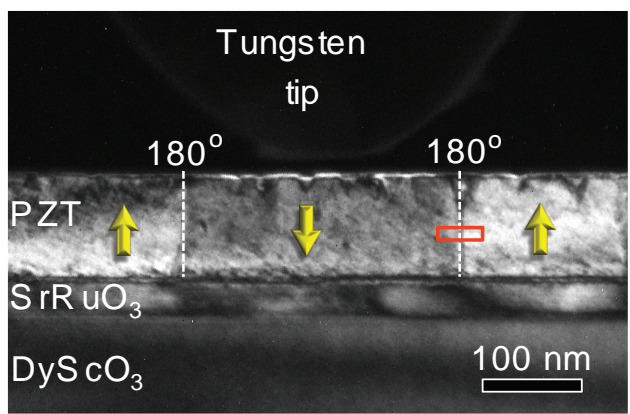

d

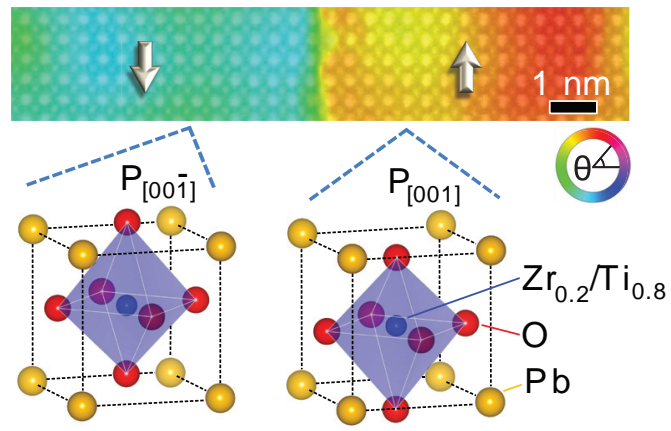

Figure 1. $180^{\circ}$ domain walls induced by external electric fields. PFM images showing $180^{\circ}$ domain walls written in lithography mode with a voltage bias of $+17.5 \mathrm{~V}$ (down) and $-17.5 \mathrm{~V}$ (up). a) The overlay of PFM phase on the amplitude, indicating a $180^{\circ}$ rotation. b) PFM amplitude image overlaid on surface topography shows the independence of the domain wall position (blue) from the surface contours. c) Dark field in situ TEM image showing the geometry of the tungsten tip-PZT-SRO heterostructure. A switched domain corresponding to a $180^{\circ}$ rotation of the polarization appears in dark contrast imaged using $g=(202)$. The polarization direction is indicated by arrows. d) A sub-Ångstom resolution HAADF image overlaid with its polarization angle, revealing a $180^{\circ}$ domain wall.

change in phase (color), but not amplitude (height), indicates a $180^{\circ}$ rotation. The film topography is overlaid on the PFM amplitude image in Figure $1 \mathrm{~b}$, which shows some roughening on film surface, however, the domain wall (blue line) does not follow the surface contours. Local nanoscale switching carried out in situ on thin TEM cross sections is also determined to be $180^{\circ}$, an example dark field image of a switched region is shown in Figure 1c. The amplitude and direction of the polarization across the $180^{\circ}$ domain wall were determined by calculating the relative offset of the $\mathrm{Zr} / \mathrm{Ti}$ atoms to the $\mathrm{Pb}$ sublattice using high-resolution high angle angular dark field (HAADF) images. ${ }^{[25]}$ Figure 1d shows the HAADF image of the switched domain wall overlaid with its atom-offset derived polarization angle, confirming a $180^{\circ}$ rotation of the polarization.

Although the as-grown PZT thin film is primarily polarized upward $\left(P_{[001]}\right)$, some downward $\left(P_{[00 \overline{1}]}\right)$ polarized regions are frequently observed. A time series of a $P_{[001]}$ domain (light) formation and subsequent backswitching from one such $P_{[00 \overline{1}]}$ polarized region (dark) under a positive-field is shown in Figure $2 \mathrm{a}-\mathrm{d}$ (see Supporting Information Video 1). The images correspond to the negative voltage cycle of a $0.1 \mathrm{~Hz}, 18 \mathrm{~V}_{\text {peak-to-peak }}$ triangular wave. The corresponding hysteresis loop is shown in Figure 2e determined by the domain volume. Formation of a $P_{\text {[001] }}$ domain occurred at $-6.6 \mathrm{~V}$ during the initial $0 \mathrm{~V}$ to $-9 \mathrm{~V}$ ramp and remained stable until $-5.5 \mathrm{~V}$ of the returning $-9 \mathrm{~V}$ to $0 \mathrm{~V}$ ramp (Figure $2 \mathrm{~b}$ ). Further reduction in the voltage resulted in the rotation of the domain walls towards the equilibrium (100) planes (vertical) as shown in Figure 2c. This leads to the annihilation of the two opposing domain walls and the complete backswitching of the $P_{[001]}$ domain (Figure 2d).

The formation of a $P_{[001]}$ domain can be stabilized by an increase in the domain size using a larger magnitude and/or longer duration of the applied voltage (see Supporting Information Video 2). A time series of the domain structure evolution during switching in the same region of the film under a larger voltage and longer dwell time is shown in Figure 3. The initial domain structure is identical (Figure 3b). However, the longer switching time allows for slow lateral expansion of the $P_{[001]}$ domain (Figure 3c-d). Upon removal of the applied voltage the relaxation of the domain walls no longer brings them into contact (Figure 3e), but rather results in only a reduction in domain size as the domain walls acquire typical equilibrium vertical $180^{\circ}$ domain orientations (Figure $3 \mathrm{f}$ ).

According to first-principles calculations the $180^{\circ}$ domain walls in PZT along the low energy $\{100\}$ planes have energy densities of about $130 \mathrm{~mJ} / \mathrm{m}^{2}$. $^{[17]}$ However, the highly inclined orientations formed under applied electric fields, observed in Figures 2 and 3, results in positive bound charges from the head-to-head orientation of the polarization vectors normal to the domain wall plane (Figure 4a). Given the domain wall incline $\theta=55^{\circ}$ from figure $2 \mathrm{~b}$, the bound charge density from the termination of each of the polarization directions at the 

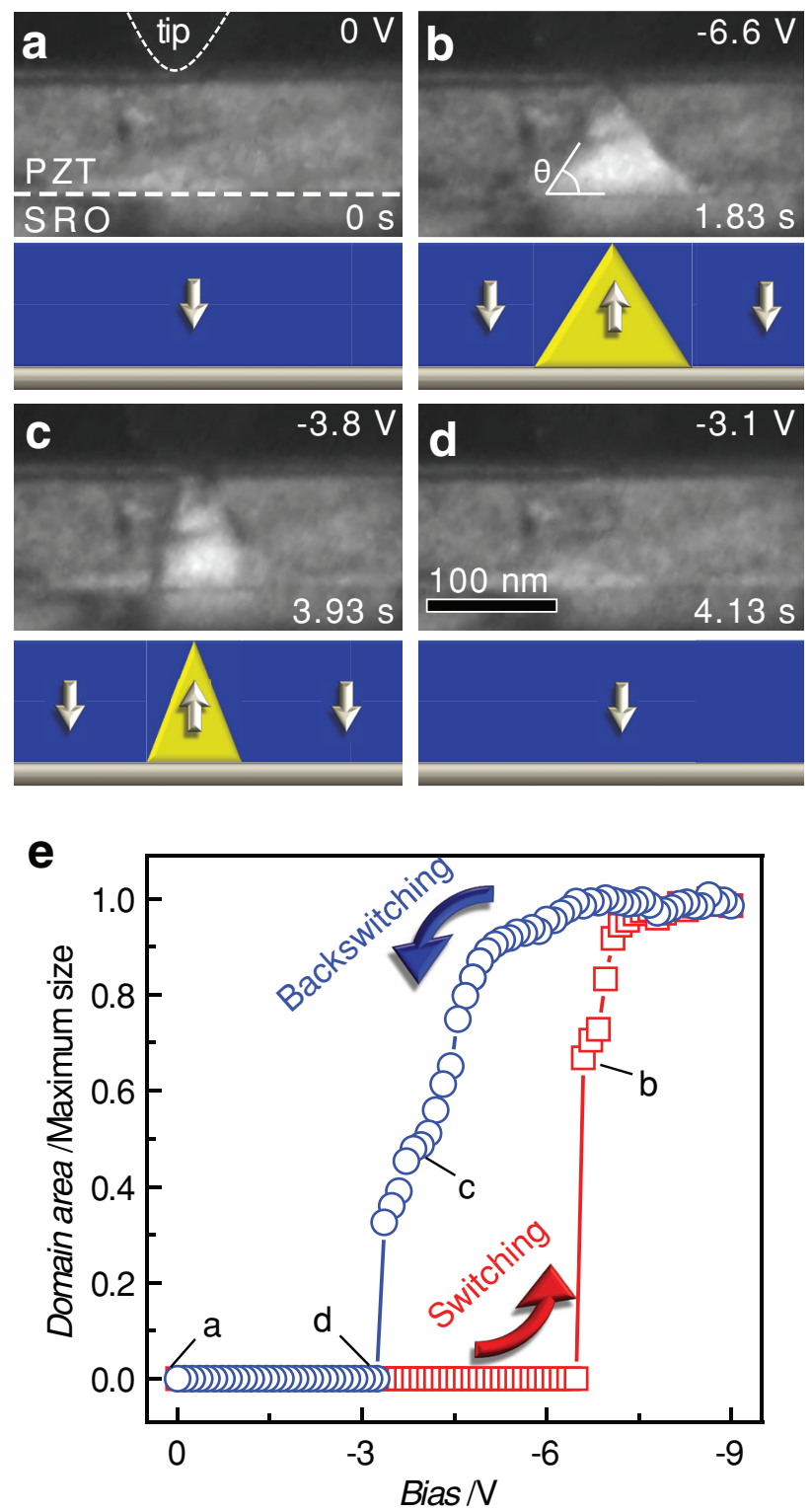

Figure 2. Polarization retention loss in a small switched domain. a-d) Continuous image sequence of domain evolution in PZT film under linear $0 \rightarrow-9 \rightarrow 0 \mathrm{~V}$ voltage ramps. The time elapsed (in seconds) and bias (in volts) are marked in the figures. a) The initial domain was polarized downward $\left(P_{[001]}\right)$. b) An upward polarized triangular shaped domain $\left(P_{[001]}\right)$ forms at $-6.6 \mathrm{~V}$. With the decrease in voltage, this domain (c) shrinks by the rotation of the inclined domain walls to their vertical equilibrium positions and (d) finally switches back completely. e) The polarization hysteresis loop calculated by the projected domain area of the $P_{[001]}$ domain as a function of the applied bias.

domain wall $\left(\cos \theta P_{S}\right)$ is $0.57 P_{S}$, where $P_{S}$ is the spontaneous polarization. This gives a total charge density of $1.14 P_{S}$ which exceeds even the surface charge density of the film $\left(P_{S}\right)$. Given an approximate value of $P_{S}=70 \mu \mathrm{C} / \mathrm{cm}^{2},{ }^{[26,27]}$ this corresponds to a charge density of $80 \mu \mathrm{C} / \mathrm{cm}^{2}$ or an equivalent density of $5.0 \cdot 10^{14} \mathrm{~cm}^{-2}$ elementary charges. This charge makes the orientation of the domain walls metastable, leading to spontaneous relaxation to $\{100\}$ planes after the bias is removed and a shrinking of the switched domain area. For small domains where the apex of these two walls meet to form a triangle shaped domain, as in Figure 4a, the backswitching leads to a complete reversal of polarization within a few seconds. Larger trapezoidal domains (Figure 4b) suffer the same immediate loss in domain volume from the domain wall reorientation, but still retain a rectangular switched domain (Figure $4 \mathrm{c}$ ). These domains still remain stable after four months $\left(10^{7}\right.$ seconds $)$, indicating thermodynamic stability.

The driving force for adopting metastable domain orientations can be understood from the electric field distribution within the PZT film. The two primary contributions are the Schottky junction at the PZT/SRO interface (Figure 4d) and the external field applied between the surface probe and SRO electrode (Figure 4e). The former arises from the larger work function of SRO $(4.9 \mathrm{eV})$ in comparison with the electron affinity of PZT $(3.5 \mathrm{eV}) .{ }^{[28,29]}$ The resulting electric field is normal to the interface and increases linearly with depth, reaching its maximum value $\sim 18 \mathrm{MV} / \mathrm{m}$ at the PZT/SRO interface ${ }^{[30]}$ as shown in the contour plot (Figure 4d), derived assuming homogenous doping and a depletion width equal to the film height. In contrast, the external applied field is concentrated at the top near the film surface due to the local contact of the tip. The inhomogeneous vertical electric field distribution is shown in Figure 4e for a $-6 \mathrm{~V}$ bias and $10 \mathrm{~nm}$ tip contact area. The combination of these two fields produces the distribution of the total field shown in Figure 4f. The equipotential lines in this contour map exhibit the same triangular shape for moderate field strengths (Figure 4f) as the initial switched domain. Since the domain nucleation and domain wall motion are field dependent, new domains will nucleate from one of the two maximum field regions at the top and bottom interfaces, expand rapidly through high field regions (red), and slow down as they extend to regions of low field resulting in inclined orientations. Lateral growth of the domain away from the tip would be particularly slow at the top surface where the applied field is almost entirely in plane. Similar switching experiments in the opposite direction, forming $P_{[00 \overline{1}]}$ domains within a $P_{[001]}$ region, exhibited very little inclination of the domain walls from the stable vertical planes and backswitching by this mechanism was not observed.

Although the applied electric field and the Schottky barrier between PZT and SRO can account for a tapered domain shape, other possible built-in fields may be present and destabilize the $P_{[001]}$ domain near the free surface. These fields may result from a Schottky junction between the tungsten tip and $\mathrm{PZT}^{[28,31]}$ or from the possible diffusion of charged defects and adsorption of charged species aligned to the original $P_{[00 \overline{1}]}$ orientation. The presence of surface states and the short contact of the tungsten tip to the PZT film at room temperature, which is unlikely to have reached electrochemical equilibrium, makes the W/PZT Schottky field difficult to estimate. The built-in field at this junction has the potential to be slightly larger than at the PZT/SRO interface, but opposite in direction, reducing the applied field at the tip contact point. The contribution of the alignment of defect dipoles to the original $P_{[00 \overline{1}]}$ polarization, an aging process common to ferroelectric 

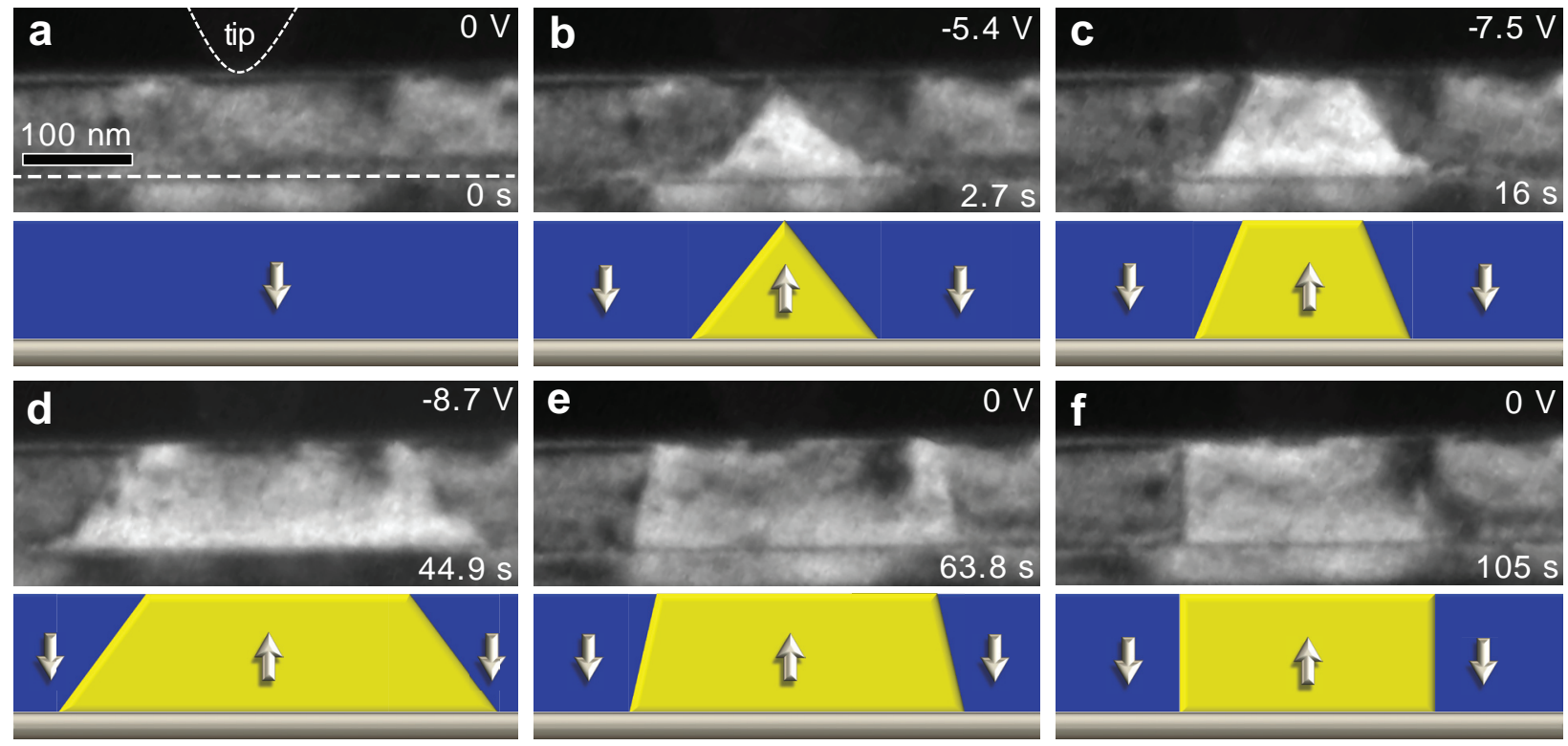

Figure 3. Partial retention loss. a) Original polar state is downward. b) A triangular $P_{[001]}$ domain is created at $-5.4 \mathrm{~V}$ bias with inclined domain walls. c-d) With increase of the amplitude and duration of the voltage, the domain laterally expands into a final trapezoidal shape. e) The inclined domain walls relax after removal of the bias reducing the switched area and forming, f) a rectangular stable domain.

oxides, must be either sufficiently weak or rapidly reversed so that the induced $P_{[001]}$ orientation is thermodynamically stable as evidenced by the long term retention of all but the smallest domains.

In conclusion, given the positive field at the PZT/SRO Schottky junction and the lack of strain in $180^{\circ}$ switching, the induced $P_{[001]}$ domain may not only be degenerate, but thermodynamically favored in the film at zero bias. In such a case the backswitching of small domains can be solely attributed to the relaxation of their high-energy domain walls. Practically this imposes a critical size criteria on the formation of stable switched domains, similar to the critical size for domain nucleation. This also reveals that for a ferroelectric thin film in an inhomogeneous field lateral domain wall creep does not take place by the stepwise movement of equilibrium domain walls, as it is typically modeled. ${ }^{[32]}$ The propagation of charged, rather than neutral domain walls may significantly alter the kinetics of the switching since the domain wall itself will electrostatically
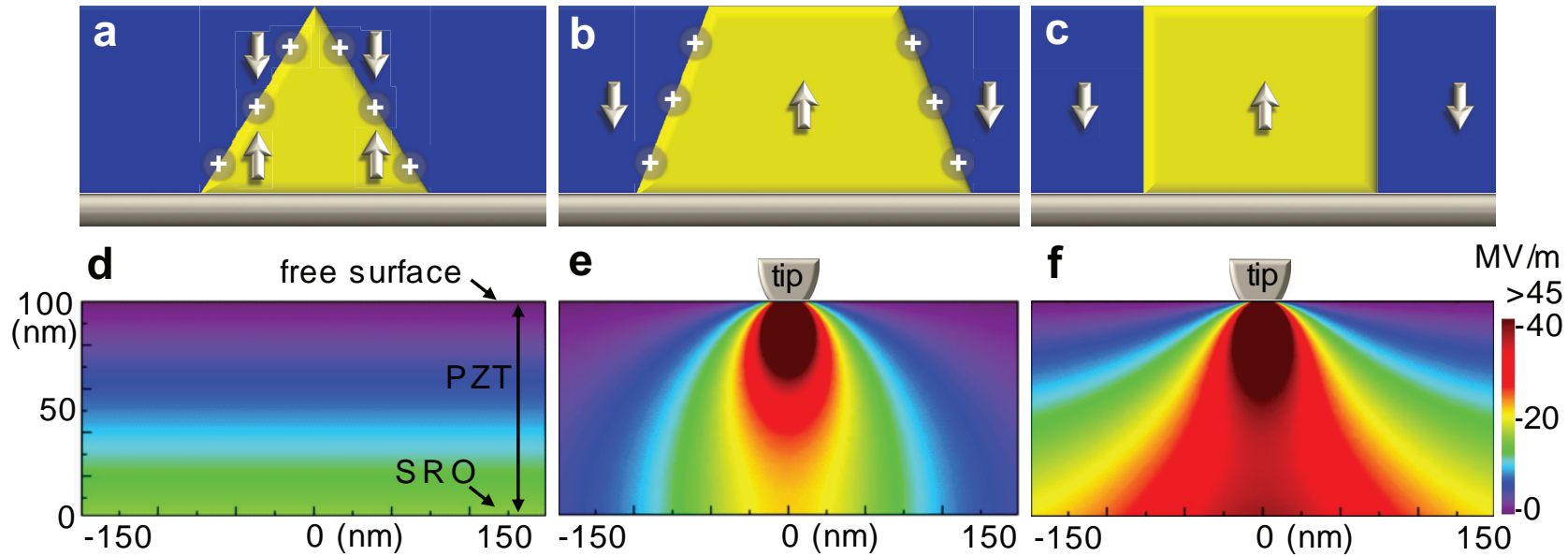

Figure 4. Domain shape and electric field distribution. Inclined domain walls in (a) triangular and (b), trapezoidal domains are positively charged due to head-to-head polarization arrangements. c) At zero bias stable domains are rectangular with uncharged vertical domain walls. d) Contour plot showing the distribution of the built-in electric field $\left(E_{b i}\right)$ in a fully depleted PZT film resulting from a Schottky barrier between PZT and SrRuO $\mathrm{O}_{3}$. $E_{b i}$ decreases linearly from its maximum value $(\sim 18 \mathrm{MV} / \mathrm{m})$ at the interface toward zero at the top surface. e) Contour plot of the vertical component of the external electric field $\left(E_{\text {ext }}\right)$ in PZT calculated with a tip contact width of $10 \mathrm{~nm}$ and voltage $-6 \mathrm{~V}$. $\left.\mathrm{f}\right)$ The combination of these electric fields results in the contour plot with tapered lines of equipotential field strength, the bright red region corresponding closely to the triangular domain shape initially adopted during switching. 
interact with charged defects and built in fields. The dynamic variation of the domain wall along the film depth is an important consideration not only for ferroelectric storage, but for surface probe microscopy which utilizes this same geometry.

\section{Experimental Section}

Materials synthesis and characterization: Epitaxial $\mathrm{PbZr}_{0.2} \mathrm{Ti}_{0.8} \mathrm{O}_{3}$ and $\mathrm{SrRuO}_{3}$ films were grown on single crystal (110) $\mathrm{DyScO}_{3}$ substrates by $90^{\circ}$ off-axis sputtering. SRO was grown at $630^{\circ} \mathrm{C}$ and $200 \mathrm{mTorr}$ with $3: 2$ ratio of $\mathrm{Ar}$ to $\mathrm{O}_{2}$ and PZT at $530{ }^{\circ} \mathrm{C}$ and 200 mTorr with a 1:1 ratio of $\mathrm{Ar}$ to $\mathrm{O}_{2}$ gasses. Cross-sectional TEM specimens were prepared by mechanical polishing followed by argon ion milling. In situ TEM experiments were carried out using JEOL 2010F, 3011 and the spherical aberration (Cs) corrected $2100 \mathrm{~F}$ microscopes equipped with a Nanofactory scanning tunneling microscopy (STM) system. A bias voltage was applied between an electrochemically etched tungsten tip, serving as a top electrode, and the conductive SRO bottom electrode which was connected to the holder ground. External voltages were applied using an HP 6614C power supply for DC voltages and an HP 3324A function generator. The domain structure was imaged in real time using diffraction contrast imaging and recorded by a TV-rate camera at 30 frames per second. Polarization directions were determined by scanning transmission electron microscope (STEM) using a Cs corrected FEl Titan 80-300 operated at $300 \mathrm{kV}$ (TEAM 0.5) and a Cs corrected JEOL 2100F.

Piezoresponse force microscopy (PFM): PFM experiments were carried out on an Asylum Research MFP-3D AFM using Umasch NSC18/Pt tips tuned to resonance with the sample. Sample poling was performed in lithography mode, with an applied voltage of $+/-17.5 \mathrm{~V}$.

Electric field distribution: $A$ linear built-in electrical field $\left(E_{b i}\right)$ from the Schottky barrier between SRO and PZT is used in this work, assuming homogenous space charge, i.e., homogenous doping, vacancies, and charge injection (or a lack thereof). The maximum $E_{b i}$ value of $\sim 18 \mathrm{MV} / \mathrm{m}$ at the interface of SRO/PZT is derived from estimated work functions of $5.8 \mathrm{eV}$ for PZT and $4.9 \mathrm{eV}$ for SRO neglecting interface states. The external electrical field $\left(E_{\text {ext }}\right)$ was modeled by finite element calculations using Ansoft Maxwell software assuming tungsten as a perfect conductor and PZT as an insulator with a resistivity of $10^{7} \Omega \mathrm{m}$ and a relative dielectric constant of 1000 . The tip contact width was estimated to be $\sim 10 \mathrm{~nm}$.

\section{Supporting Information}

Supporting Information is available from the Wiley Online Library or from the author.

\section{Acknowledgements}

This work at the University of Michigan was supported by the Department of Energy (DOE) under the grant DE-FG02-07ER46416 and partially by the National Science Foundation under grants DMR-0907191 (Y.Z.), DMR-0820404 (JRJ) and DMR-0723032 (TEM instrument). The work at University of Wisconsin-Madison was supported by the Army Research Office under Grant No. W911NF-10-1-0362. The authors would also like to acknowledge the National Center for Electron Microscopy at Lawrence Berkeley National Laboratory for their support under the DOE grant DE-AC02-05CH11231 for user facilities.

Received: October 17, 2011 Revised: December 14, 2011
[1] J. F. Scott, C. A. P. Dearaujo, Science 1989, 246, 1400.

[2] O. Auciello, J. F. Scott, R. Ramesh, Phys. Today 1998, 51, 22.

[3] J. F. Scott, Science 2007, 315, 954.

[4] R. Ramesh, N. A. Spaldin, Nat. Mater. 2007, 6, 21.

[5] O. Lohse, M. Grossmann, U. Boettger, D. Bolten, R. Waser, J. Appl. Phys 2001, 89, 2332.

[6] A. Gruverman, M. Tanaka, J. Appl. Phys. 2001, 89, 1836.

[7] C. S. Ganpule, A. L. Roytburd, V. Nagarajan, B. K. Hill, S. B. Ogale, E. D. Williams, R. Ramesh, J. F. Scott, Phys. Rev. B 2002, 65, 014101.

[8] H. Y. Guo, J. B. Xu, I. H. Wilson, Z. Xie, E. Z. Luo, S. B. Hong, H. Yan, Appl. Phys. Lett. 2002, 81, 715.

[9] B. S. Kang, D. J. Kim, J. Y. Jo, T. W. Noh, J. G. Yoon, T. K. Song, Y. K. Lee, J. K. Lee, S. Shin, Y. S. Park, Appl. Phys. Lett. 2004, 84, 3127.

[10] D. J. Kim, J. Y. Jo, Y. S. Kim, Y. J. Chang, J. S. Lee, J. G. Yoon, T. K. Song, T. W. Noh, Phys. Rev. Lett. 2005, 95, 237602.

[11] J. M. Benedetto, R. A. Moore, F. B. McLean, J. Appl. Phys. 1994, 75, 460.

[12] G. Arlt, H. Neumann, 1988, 87, 109

[13] I. P. Batra, B. D. Silverman, Solid State Commun. 1972, 11, 291.

[14] R. R. Mehta, B. D. Silverman, J. T. Jacobs, J. Appl. Phys. 1973, 44, 3379.

[15] M. P. Cruz, Y. H. Chu, J. X. Zhang, P. L. Yang, F. Zavaliche, Q. He, P. Shafer, L. Q. Chen, R. Ramesh, Phys. Rev. Lett. 2007, 99, 217601.

[16] S. H. Baek, H. W. Jang, C. M. Folkman, Y. L. Li, B. Winchester, J. X. Zhang, Q. He, Y. H. Chu, C. T. Nelson, M. S. Rzchowski, X. Q. Pan, R. Ramesh, L. Q. Chen, C. B. Eom, Nat. Mater. 2010, 9, 309.

[17] B. Meyer, D. Vanderbilt, Phys. Rev. B 2002, 65, 11.

[18] A. Lubk, S. Gemming, N. A. Spaldin, Phys. Rev. B 2009, 80, 8.

[19] C. B. Eom, R. J. Cava, R. M. Fleming, J. M. Phillips, R. B. Vandover, J. H. Marshall, J. W. P. Hsu, J. J. Krajewski, W. F. Peck, Science 1992, 258, 1766.

[20] C. B. Eom, R. B. Vandover, J. M. Phillips, D. J. Werder, J. H. Marshall, C. H. Chen, R. J. Cava, R. M. Fleming, D. K. Fork, Appl. Phys. Lett. 1993, 63, 2570.

[21] M. Dawber, K. M. Rabe, J. F. Scott, Rev. Mod. Phys. 2005, 77, 1083.

[22] C. T. Nelson, P. Gao, J. R. Jokisaari, C. Heikes, C. Adamo, A. Melville, S. H. Baek, C. M. Folkman, B. Winchester, Y. J. Gu, Y. M. Liu, K. Zhang, E. G. Wang, J. Y. Li, L. Q. Chen, C. B. Eom, D. G. Schlom, X. Q. Pan, Science 2011, 334, 968.

[23] H. J. Chang, S. V. Kalinin, S. Y. Yang, P. Yu, S. Bhattacharya, P. P. Wu, N. Balke, S. Jesse, L. Q. Chen, R. Ramesh, S. J. Pennycook, A. Y. Borisevich, J. Appl. Phys. 2011, 110, 052014.

[24] J. X. Zhang, B. Xiang, Q. He, J. Seidel, R. J. Zeches, P. Yu, S. Y. Yang, C. H. Wang, Y. H. Chu, L. W. Martin, A. M. Minor, R. Ramesh, Nat. Nanotechnol. 2011, 6, 97.

[25] C. T. Nelson, B. Winchester, Y. Zhang, S. J. Kim, A. Melville, C. Adamo, C. M. Folkman, S. H. Baek, C. B. Eom, D. G. Schlom, L. Q. Chen, X. Q. Pan, Nano Lett. 2011, 11, 828.

[26] V. Nagarajan, S. Prasertchoung, T. Zhao, H. Zheng, J. Ouyang, R. Ramesh, W. Tian, X. Q. Pan, D. M. Kim, C. B. Eom, H. Kohlstedt, R. Waser, Appl. Phys. Lett. 2004, 84, 5225.

[27] V. Nagarajan, A. Roytburd, A. Stanishevsky, S. Prasertchoung, T. Zhao, L. Chen, J. Melngailis, O. Auciello, R. Ramesh, Nat. Mater. 2003, 2, 43.

[28] J. F. Scott, Jpn. J. Appl. Phys., Part 1 1999, 38, 2272.

[29] A. J. Hartmann, M. Neilson, R. N. Lamb, K. Watanabe, J. F. Scott, Appl. Phys. A 2000, 70, 239.

[30] S. M. Sze, D. J. Coleman, A. Loya, Solid State Electron. 1971, 14, 1209.

[31] E. W. Muller, J. Appl. Phys. 1955, 26, 732.

[32] Y. H. Shin, I. Grinberg, I. W. Chen, A. M. Rappe, Nature 2007, 449, 881. 